\title{
Personal Navigation via High-Resolution Gait-Corrected Inertial Measurement Units
}

\author{
Özkan Bebek, Member, IEEE, Michael A. Suster, Student Member, IEEE, Srihari Rajgopal, Student Member, IEEE, \\ Michael J. Fu, Student Member, IEEE, Xuemei Huang, M. Cenk Çavuşoğlu, Senior Member, IEEE, \\ Darrin J. Young, Member, IEEE, Mehran Mehregany, Senior Member, IEEE, \\ Antonie J. van den Bogert, and Carlos H. Mastrangelo, Member, IEEE
}

\begin{abstract}
In this paper, a personal micronavigation system that uses high-resolution gait-corrected inertial measurement units is presented. The goal of this paper is to develop a navigation system that uses secondary inertial variables, such as velocity, to enable long-term precise navigation in the absence of Global Positioning System (GPS) and beacon signals. In this scheme, measured zerovelocity duration from the ground reaction sensors is used to reset the accumulated integration errors from accelerometers and gyroscopes in position calculation. With the described system, an average position error of $\mathbf{4} \mathbf{m}$ is achieved at the end of half-hour walks.
\end{abstract}

Index Terms-Dead reckoning, inertial measurement, Kalman filter (KF), pedestrian navigation system, pressure sensor array.

\section{INTRODUCTION}

W E ARE developing a personal micronavigation system that uses high-resolution gait-corrected inertial measurement units (IMUs). The system combines a commercial off-the-shelf (COTS) IMU with a high-resolution thin flexible error-correcting biomechanical ground reaction sensor cluster (GRSC) that is connected to a handheld processing and readout unit. The final sensor parts, including the IMU and the GRSC, will be placed within the heel and at the sole of a personnel boot and wirelessly connected to a handheld unit, which will process the data in real time. In this approach, the

Manuscript received September 11, 2009; revised November 16, 2009; accepted December 6, 2009. Date of publication April 19, 2010; date of current version October 13, 2010. This work was supported in part by the Micro Inertial Navigation Technology Program of the Defense Advanced Research Projects Agency (DARPA). The views, opinions, and/or findings contained in this article/presentation are those of the author/presenter and should not be interpreted as representing the official views or policies, either expressed or implied, of the DARPA or the Department of Defense. Approved for public release, distribution unlimited. The Associate Editor coordinating the review process for this paper was Dr. Salvatore Baglio.

Ö. Bebek, M. A. Suster, S. Rajgopal, M. J. Fu, and M. Mehregany are with the Department of Electrical Engineering and Computer Science, Case Western Reserve University, Cleveland, OH 44106 USA (e-mail: oxb6@ case. edu; mas20@case.edu; sxr61@case.edu; mjf24@case.edu; mehran@case.edu).

X. Huang and A. J. van den Bogert are with the Department of Biomedical Engineering, Lerner Research Institute, Cleveland Clinic, Cleveland, OH 44195 USA (e-mail: huangx2@ccf.org; bogert@bme.ri.ccf.org).

M. C. Çavuşoğlu is with the Department of Electrical Engineering and Computer Science, Case Western Reserve University, Cleveland, OH 44106 USA, and also with the Department of Electrical and Electronic Engineering, Bilkent University, Ankara 06800, Turkey (e-mail: cavusoglu@ case.edu).

D. J. Young and C. H. Mastrangelo are with the Department of Electrical and Computer Engineering, University of Utah, Salt Lake City, UT 84112 USA (e-mail: darrin.young@utah.edu; mastrang@ece.utah.edu).

Color versions of one or more of the figures in this paper are available online at http://ieeexplore.iee.org.

Digital Object Identifier 10.1109/TIM.2010.2046595

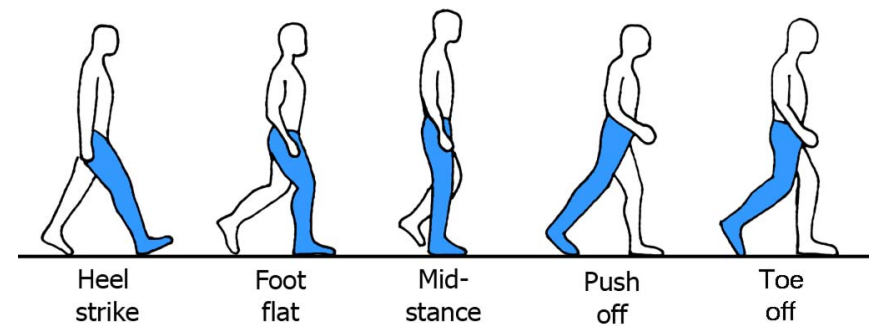

Fig. 1. Stance phase in human bipedal locomotion. In this phase, the foot is in contact with the ground. Only at specific times, during the midstance, the foot heel remains stationary (figure modified from [14]).

IMU will measure inertial displacements, and the GRSC will independently measure dynamic ground forces, shear strains, and sole deformation associated with a ground locomotion gait. The high-resolution biomechanical GRSC data can be used to accurately detect periods of zero velocity. These zero-velocity points provide discrete zero-velocity corrections to the IMU that dramatically increase its effective positioning resolution.

Step-corrected (also known as dead reckoning) IMU and GPS navigation systems have been in existence for several years [1]-[12]; however, unlike our proposed approach, these systems detect the step impact shock with accelerometers that are placed away from the ground. This approximate detection technique typically results in a $1 \%-2 \%$ positioning error. In our approach, we use a data-rich high-resolution GRSC that is placed very close to the point of heel-to-ground contact to provide detailed contact information to an IMU that is located in close proximity to the GRSC. We believe that this extra information and the close mechanical (near rigid) relation between the velocity at the GRSC and IMU locations [13] are key to reach the high-resolution positioning improvements. Our goal with this unique sensor data fusion approach is to ultimately permit accurate navigation on any indoor or outdoor terrain, unassisted by external signals.

In human bipedal locomotion, the walking mode or gait consists of two separate phases [14]. In the swing phase, the leg is off the ground. This period extends from the instant the toe leaves the ground until the heel strikes. In the stance phase (Fig. 1), the foot heel first contacts the ground, and then it rolls until the midstance is reached resulting in pivoting of the leg on the ankle (and the corresponding forward motion of the body). Beyond the midstance, detachment of the foot takes place through gradual rolling. It is evident that only during a fraction of the midstance the velocity of the heel is exactly zero [15]. 

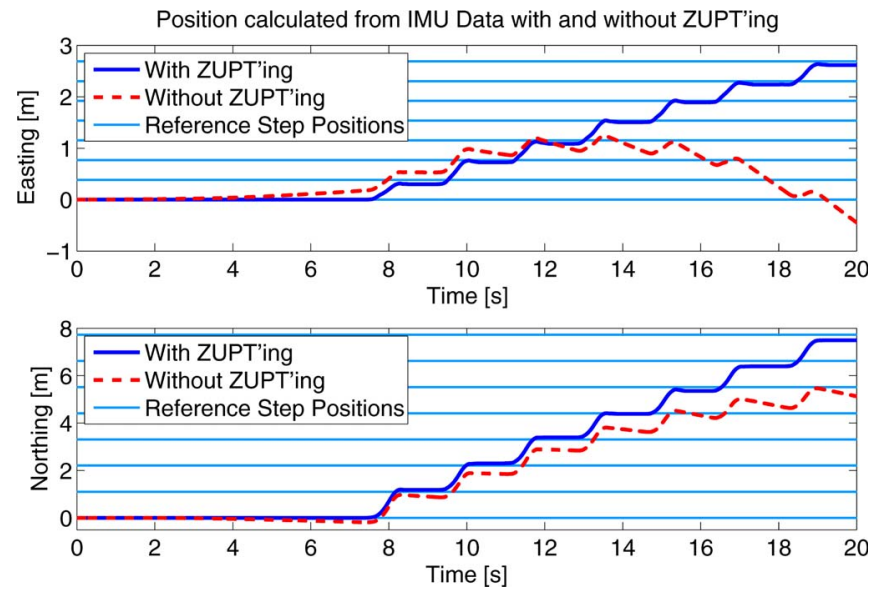

Fig. 2. Comparison of position calculations with and without ZUPT'ing. The user walks on a straight line headed toward northeast. The figure shows the position of the user with respect to time, where Easting refers to the eastwardmeasured distance, and Northing refers to the northward-measured distance. Foot-stepping positions are shown with horizontal grid lines. Dashed lines in the figure indicate the drifted position. Drifting errors build up in a few seconds without zero-velocity correction.

Hence, we propose to detect this time period very precisely with the GRSC. A high-density GRSC can detect very small changes in the stationary contact yielding very small errors in the velocity determination in the stance phase. Relating the velocity of the rolling contact to the heel velocity, where the IMU is located, can provide us to detect zero-velocity points.

Our initial design goals for the personal micronavigation system are as follows: 1) navigation accuracy below $10 \mathrm{~m}$ for half-hour walking; 2) velocity sensing bias per step below $4 \mathrm{~mm} / \mathrm{s}$; 3) maximum volume for the GRSC below $10 \mathrm{~cm}^{3}$; 4) power consumption of the GRSC (including the transmission system) below $300 \mathrm{~mW}$; and 5) a GRSC with at least ten sensor elements. If these design goals are met, the developed system will exceed the current state-of-the-art micronavigation systems.

The navigation accuracy goals were set relative to the state of the art, $45 \mathrm{~m}$ in one-half hour projected from a shorter walk [10]. The velocity sensing bias of $4 \mathrm{~mm} / \mathrm{s}$ was chosen as the minimum detectable velocity threshold that could be verified experimentally. Based on this sensing bias, and the heel contact area as the sensing area, the minimum number of sensor elements was determined. The volume was chosen to fit the GRSC under the shoe insole. The power consumption of $300 \mathrm{~mW}$ was chosen to enable selection from a wide spectrum of potential COTS GRSC technologies.

\section{NAVIGATION WITH THE IMU AND ZUPT'ING}

The basic idea behind a gait-corrected navigation system is to use the walking stride to periodically reset the drifting IMU, thus dramatically reducing cumulative navigation errors. The correction occurs when the foot is on the ground, when all velocities and accelerations of the shoe are zero. Without this correction, even the smallest measurement errors, due to sensor drift or sensor noise, will amplify and cause drifting errors of a few meters in four to five steps. Fig. 2 compares the position that is calculated by integrating the acceleration twice with zero-velocity updating (ZUPT'ing) during the stance period and the position that is calculated without any velocity updates.

The effectiveness of stance-based ZUPT'ing depends on the detection of zero velocity at the stance period. Most personal dead-reckoning (PDR) systems detect steps using a pedometer or an accelerometer and move the position estimate forward by the step length in the direction that is determined by a magnetic compass or a yaw gyroscope [6], [7], [16]-[18]. The sensors that are attached to the upper body detect motion from normal acceleration or phasing acceleration axes that exhibit cycles that are typical of a human's walking motion. The number of steps is counted in a pedometer system. The average step length is adjusted for the walking speed and then used to calculate the user's position. More sophisticated systems analyze the accelerometer signals to estimate step lengths. All of these systems require calibration to an individual user because everyone's gait has different acceleration profiles. An inertial navigation system embedded in a soldier's boot heel is described by Elwell [19], but no experimental validation was performed. Stirling et al. [20] describe an experiment using a prototype shoe-mounted sensor that measures stride length with accelerometers and direction with magnetometers. The system measures angular acceleration using pairs of accelerometers. The system stops integrating and resets the velocity before each step. Errors up to $20 \%$ of distance traveled were reported.

A more complex pedometer-like approach was introduced by Cho and Park [21]. Their system uses a two-axis accelerometer and a two-axis magnetometer located on a shoe. Step length is estimated from accelerometer readings that are passed through a neural network, and a Kalman filter (KF) was used to reduce magnetic disturbances. Although their outdoor results are good, they could not filter the magnetic disturbances well indoors, which resulted in large errors. A fiducial-based position estimation system was proposed by Saarinen et al. [22]. Ultrasonic sensors attached to boots were used to measure the length of every stride in real time. In straight-line walking experiments, the authors report an average and a maximum error of $1.3 \%$ and $5.4 \%$, respectively. Another fiducial-based approach introduced by Brand and Phillips [23] uses radio frequency phase changes between a reference signal that is located in a waist pack and the one coming from a transmitter that is located on each boot. This system's measurements are limited to 2-D environments and cannot detect altitude changes.

Recently, Ojeda and Borenstein [10] and Borenstein et al. [12] have developed a shoe-based navigation system that uses a small six-degree-of-freedom (6-DOF) IMU that is attached to a user's boot. The IMU provides rate-of-rotation and acceleration measurements that are used in real time to estimate the location of the user relative to a known starting point. To reduce the most significant errors of this IMU-based system, they used ZUPT'ing. With the ZUPT technique and related signal processing algorithms, the relative error of the system was about $2 \%$ of the distance traveled. In this typical PDR system, the error is independent of the gait or the speed of the user. Their PDR system works in 3-D environments, although errors in the $z$-direction are usually larger than $2 \%$ of the distance traveled. Feliz et al. [24] used an IMU and a GPS and barometer unit in their PDR system. They did short indoor and outdoor walks to 
test the system. Their best relative position loop-closing errors were around $2 \%$ for outdoor walks and $10 \%$ for indoor walks.

Many of the PDR devices attempt to perform the ZUPT'ing by detection of the contact of the foot with the ground. Most stance-based schemes in the literature equate zero-velocity detection to the impact of the heel when it hits the ground. The problem with this scheme is that the impact shock event only signals the beginning of the stance phase, which involves several subphases itself. Not all subphases have zero velocity. Zero velocity only occurs at some point around the midstance subphase, after all rolling contact of the foot with the ground has been reached. If the zero-velocity point is not accurately determined, the resulting ZUPT'ing scheme will have an intrinsic zero-velocity bias that will reduce its effectiveness. To accurately detect zero-velocity stance, it is necessary to utilize a sensor at the IMU location to record sufficient data detailing the nature of the contact with the ground. In this paper, we propose to use a GRSC that is placed in close proximity to the IMU location to more accurately estimate the periods of zero velocity, improving ZUPT'ing and reducing the position error.

For initial system development, a COTS IMU and a highresolution GRSC were connected to a laptop that served as a data acquisition and integration component of the system. The collected data were processed offline. The ultimate goal is to replace the laptop with a more portable handheld device like a personal digital assistant that is capable of real-time data processing. In the following, each of the sensors that are used in the system and their integration approach is explained in detail.

\section{IMU}

We used an InterSense InertiaCube 3 module that integrates two two-axis accelerometers, three single-axis gyroscopes, and a three-axis magnetometer compass within a low volume $\left(26.2 \times 39.2 \times 14.8 \mathrm{~mm}^{3}\right)$, which can fit within the heel. This IMU module is temperature-compensated producing a lower error bias than those of other COTS IMUs. InterSense [25] has developed one of the most sophisticated extended $\mathrm{KF}$ schemes for micronavigation. InertiaCube 3 combines the aforementioned sensing elements with an integrated Kalman filtering algorithm. The unit can provide orientation and gravitycompensated acceleration information aligned with the Earth's magnetic north. InertiaCube 3 can measure accelerations up to \pm 6 g. Prior to shipment, the IMU performance was verified to specifications using a rate table. No additional calibration for individual IMU components was necessary prior to use.

The choice of the IMU module was primarily dictated by the size to be able to fit the unit inside the heel of the shoe. The IMU incorporated a sensor data fusion algorithm that helped speed up the initial sensor integration. However, this increased the unit cost. With technology maturation and successful commercialization, we anticipate unit cost to substantially decrease.

\section{Pressure Sensor}

In our walk experiments, we used a COTS pressure sensor array, i.e., DigiTacts II Array Tactile sensors manufactured by

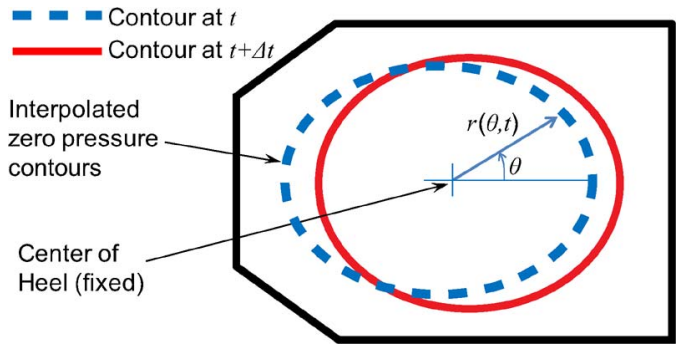

Fig. 3. Propagation of a pressure contour created by the heel.

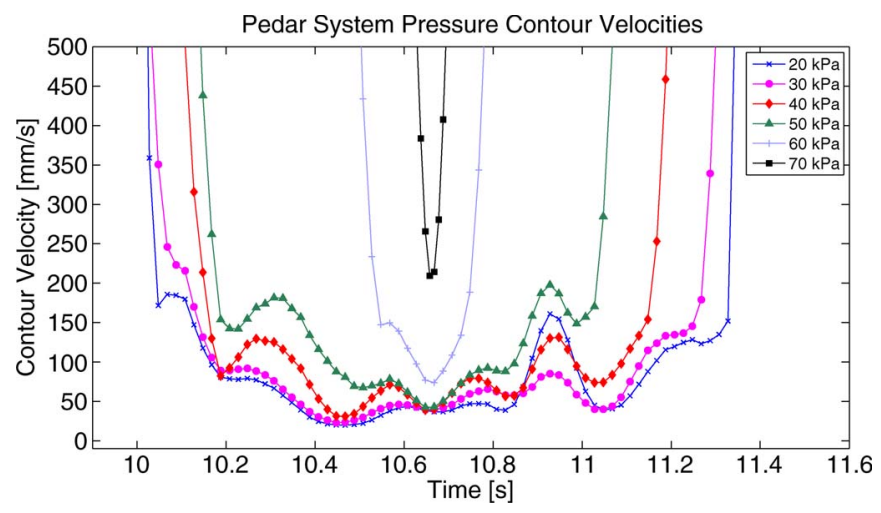

Fig. 4. Pressure contour velocities for a stance period.

Pressure Profile Systems, as our biomechanical GRSC sensor. DigiTacts II sensors have 24 sensing elements with a sensing area of $82.3 \times 45.1 \mathrm{~mm}^{2}$. These sensors use a capacitancebased sensing scheme that can detect pressures up to $140 \mathrm{kPa}$. The GRSC incorporated an $\mathrm{I}^{2} \mathrm{C}$ to a Universal Serial Bus converter that could be directly interfaced to a PC. The overall power consumption of the GRSC was $20 \mathrm{~mW}(3.3 \mathrm{~V} / 6 \mathrm{~mA})$. The GRSC was a custom-built unit that was calibrated prior to shipment.

Pressure sensor arrays can be used to detect pressure contours that are generated by the heel if the sensor is placed between the heel of the shoe and the shoe insole. One can find the centroid velocity of the pressure contours (Fig. 3) as

$$
\begin{aligned}
\vec{v}(\theta, t) & =\frac{d r(\theta, t)}{d t} \cdot \widehat{r} \approx \frac{r(\theta, t+\Delta t)-r(\theta, t)}{\Delta t} \cdot \widehat{r} \\
\vec{v}_{\mathrm{cntr}} & =\int_{0}^{2 \pi} \vec{v}(\theta, t) d \theta
\end{aligned}
$$

where $\vec{v}$ is the velocity of a point on the contour, $r$ is the radius of the contour point, $\widehat{r}$ is the unit vector that lies on the line between the center of the contour and the contour point, and $\vec{v}_{\text {cntr }}$ is the centroid velocity of the contour. Fig. 4 shows the velocities of pressure contours of the heel, i.e., $20-70 \mathrm{kPa}$ during a typical stance phase. The contour velocity can be used to detect the zero velocity of the shoe. We can assume that the shoe, and the IMU, is at rest when the centroid velocity is below a velocity threshold, i.e.,

$$
\left\|\vec{v}_{\mathrm{cntr}}\right\| \leq v_{\mathrm{thrsh}}
$$




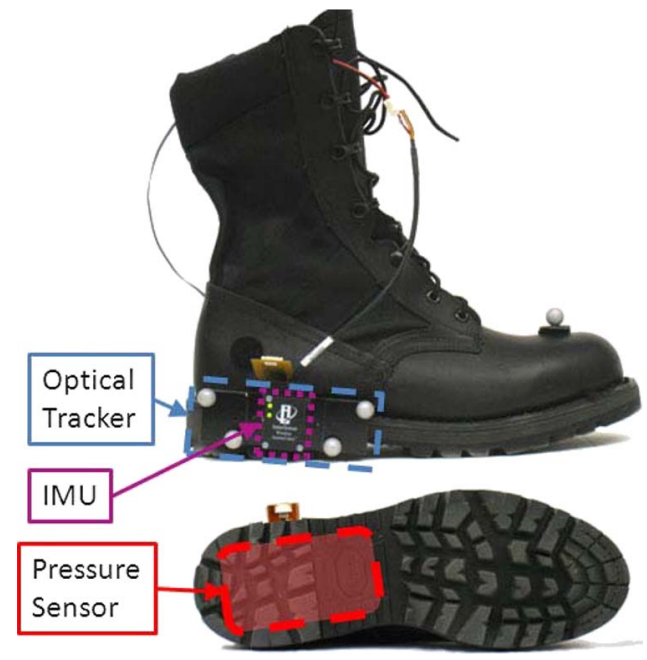

Fig. 5. Boot sensors: A boot with the pressure sensor inserted in the insole is shown. The IMU is externally located on the heel attached to an optical marker tool frame. The position and the orientation of the IMU are captured using the 3-D optical motion tracker system.

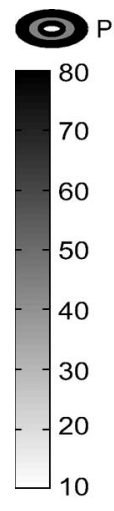

Pressure Contour $[\mathrm{kPa}]$

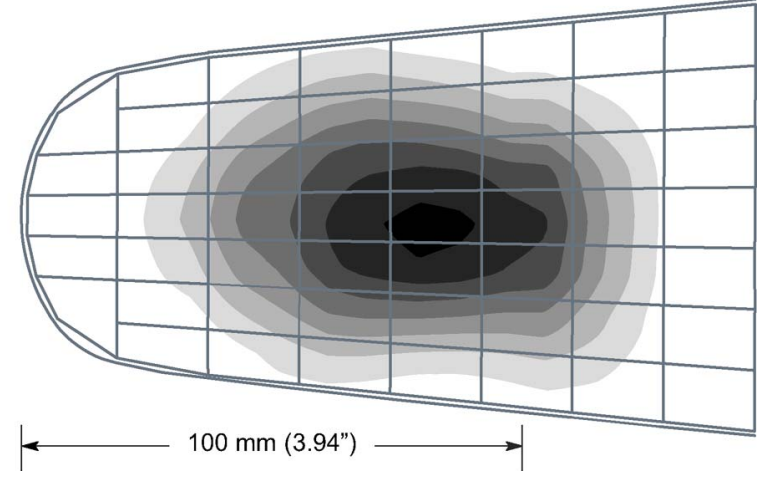

Fig. 6. Pressure contours overlaid on the heel portion of the pressure sensor insole.

This zero-velocity information can be used in the ZUPT'ing scheme to reduce the drift in the IMU measurements.

To verify that pressure sensor arrays can be used to detect zero velocities of the heel, we conducted experiments using an external sensor in addition to the IMU and the biomechanical sensor. A 3-D optical motion capture system, i.e., Eagle Digital RealTime System from Motion Analysis Corporation [26], was used as the external sensor. This sensor can provide 6-DOF position and orientation information with submillimeter rootmean-square accuracy using optical markers. The calibration of the system was performed using the procedure that is specified by the manufacturer immediately prior to data acquisition. Optical markers and an IMU were placed on a plate that was attached to the heel of a combat boot (Fig. 5). An insole-shaped pressure sensor, i.e., Novel Pedar Pressure Sensor System [27], was placed in the boot as the biomechanical sensor. This sensor has 99 sensing elements. We used 54 of the 99 elements that are located in the heel portion of the sensor (Fig. 6). Using all these sensors, walk data from each of the sensors were captured, and calculated velocity outputs were compared. Fig. 7 shows the velocity measurements from the three sensors: a boot's

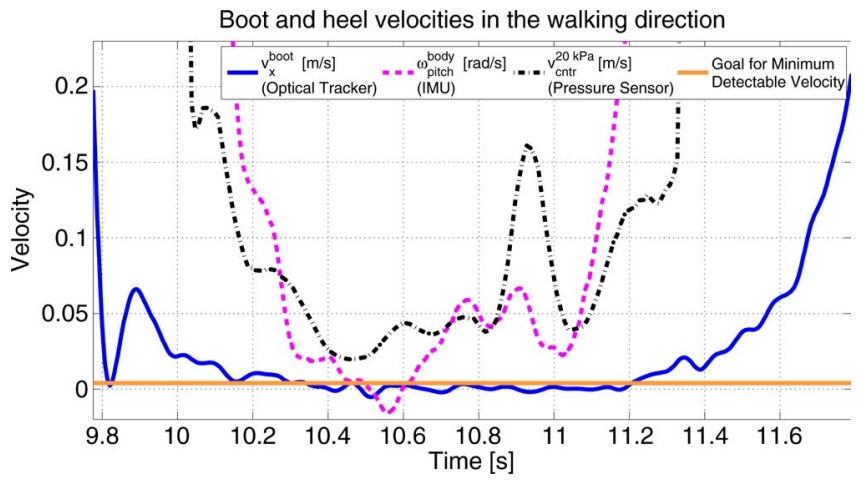

Fig. 7. Velocity measurements from the optical motion capture system, the IMU, and the pressure sensor array during a stance period. The minimum detectable velocity sensing bias goal is $4 \mathrm{~mm} / \mathrm{s}$.

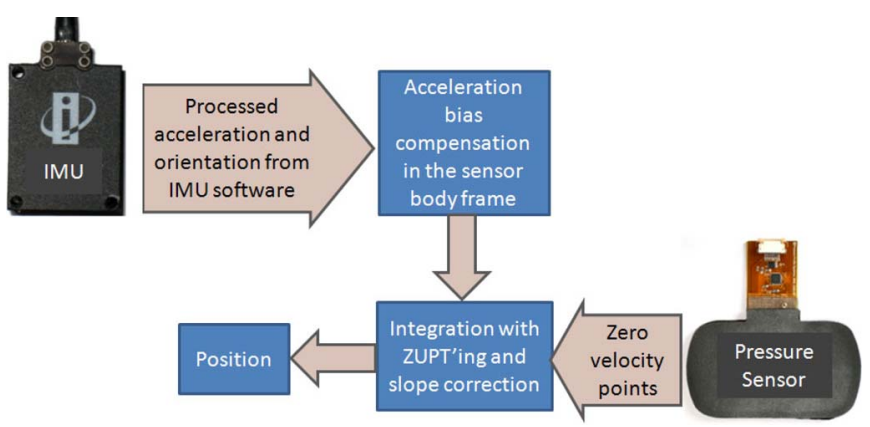

Fig. 8. Integration with the ZUPT'ing scheme followed to calculate the position from the IMU and pressure sensor outputs.

heel velocity along the walking direction, i.e., $v_{x}$, from the optical motion capture system; the angular velocity in the pitch $^{1}$ rotation, i.e., $\omega_{\text {pitch }}$, from the IMU; and the pressure contour centroid velocity of the heel, i.e., $v_{\text {cntr }}$, from the pressure system. This figure shows that the minimum detectable velocity of the boot can be observed from the pressure contour velocity of the heel, i.e., $v_{\text {cntr }}$. This provides additional and more accurate zero-velocity detection, independent of the measurements from the IMU. The optical sensor was not used in the calibration of the overall system, which is described in the latter parts of this paper.

\section{Position Calculation From the Sensors}

The sensor data fusion scheme that we followed to integrate acceleration is given in Fig. 8. First, acceleration and orientation information, which is represented in the navigation coordinate frame, ${ }^{2}$ was retrieved from the IMU. Next, a KF [29] is employed to find acceleration biases. This estimation is performed in the sensor's body coordinate frame, and then the resultant biases are transformed to the world coordinate frame. Bias-compensated acceleration from the IMU and zero-velocity points from the pressure sensor array are used in the integration

\footnotetext{
${ }^{1}$ Pitch is the measure of the rotation to which the boot's nose tilts up or down relative to its heel.

${ }^{2} \mathrm{~A}$ navigation coordinate frame or nav frame (also known as a spatial coordinate frame or a world coordinate frame) is the name that was used by the IMU manufacturer, which is the locally level geographic frame with its $x$-axis pointing north, $y$-axis pointing east, and $z$-axis pointing down [28].
} 
via ZUPT'ing to calculate the position of the user. Finally, a calibration is applied to correct the drifts in the calculated position. This calibration is used to correct future collected walk data.

In the following, the steps of this position calculation process are explained in detail.

\section{A. Acceleration Bias Compensation}

The IMU software provides gravity-compensated acceleration in the navigation coordinate frame, i.e., $a^{\text {nav }}$, and the matrix that defines the rotation between navigation and body coordinate frames, i.e., $\mathbf{R}_{\mathrm{nb}}$. Gravity-compensated acceleration in the sensor body coordinates, i.e., $a^{\text {body }}$, can be calculated as

$$
a^{\text {body }}[k]=\mathbf{R}_{\mathrm{nb}}^{-1}[k] a^{\text {nav }}[k] .
$$

Dynamics of the IMU are governed by the following equation of motion:

$$
\frac{d}{d t}\left[\begin{array}{l}
x_{s} \\
\dot{x}_{s}
\end{array}\right]=\left[\begin{array}{ll}
0 & \mathbf{I} \\
0 & 0
\end{array}\right]\left[\begin{array}{l}
x_{s} \\
\dot{x}_{s}
\end{array}\right]+\left[\begin{array}{l}
0 \\
\mathbf{I}
\end{array}\right] a_{a}^{\text {nav }}
$$

where

$$
a_{a}^{\text {nav }}=a_{m}^{\text {nav }}+a_{b}^{\text {nav }}+a_{n}^{\text {nav }} .
$$

Here, $x_{s}$ is the sensor position that is measured in the navigation frame, $a_{m}^{\text {nav }}$ is the measured acceleration in the navigation frame, $a_{b}^{\text {nav }}$ is the acceleration bias in the navigation frame, $a_{n}^{\text {nav }}$ is the zero-mean noise acceleration in the navigation frame, and $a_{a}^{\text {nav }}$ is the actual acceleration that is represented in the navigation frame that exists on the heel.

We fine-tuned the IMU measurements via a bias estimator. $\mathrm{KF}$ formulation from [29] is used to drive the estimator equations.

During the zero-velocity region of the stance period, i.e., $\dot{x}_{s}=0$, the actual acceleration of the IMU is zero, i.e., $a_{a}^{\text {nav }}=$ 0 . Then, from (6), we can write the following measurement equality in the body coordinates:

$$
-a_{m}^{\text {body }}=a_{b}^{\text {body }}+a_{n}^{\text {body }} .
$$

Consider a dynamic plant

$$
\dot{z}=\mathbf{A} z+\mathbf{B} u+\mathbf{B}_{1} w
$$

with measurements

$$
y=\mathbf{C} z+v
$$

where the process noise $w$ and the measurement noise $v$ are random sequences with zero means and have covariances $\boldsymbol{\Sigma}_{w}$ and $\boldsymbol{\Sigma}_{v}$, respectively.

Let $z \equiv a_{b}^{\text {body }}$. Assuming a constant bias, i.e., $\dot{z}=0$, we can rewrite the state-space equations as

$$
\dot{z}=\underbrace{[\mathbf{0}] z}_{\mathbf{A}} z+\underbrace{[\mathbf{0}]}_{\mathbf{B}} u+\underbrace{[\mathbf{0}]}_{\mathbf{B}_{1}} w
$$
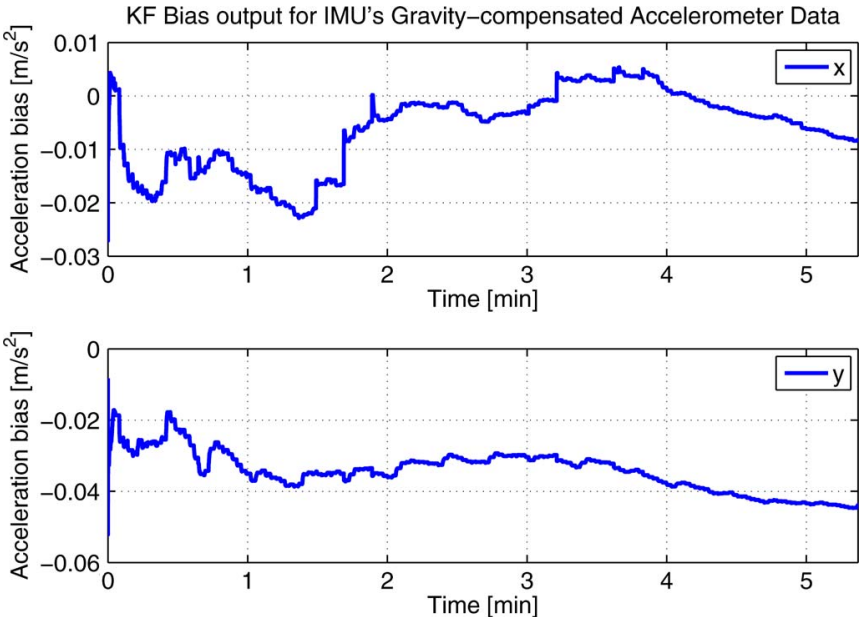

Fig. 9. Kalman filter bias output for the IMU's acceleration outputs.

and using (7), the output equation during the measurement update (where $a_{a}^{\text {body }}=0$ ) is

$$
-a_{m}^{\text {body }}=y_{2}=\underbrace{[\mathbf{I}] z}_{\mathbf{C}} z+a_{v}^{\text {body }} .
$$

For the discrete equivalent of the dynamic plant

$$
z[k+1]=\mathbf{F} z[k]+\mathbf{G} u[k]+\mathbf{G}_{1} w[k]
$$

with measurements

$$
y[k]=\mathbf{H} z[k]+v[k]
$$

the following equations can be used to estimate the acceleration bias in the body coordinates during zero-velocity periods-measurement update (during the zero-velocity region of the stance period):

$$
\hat{z}[k]=\bar{z}[k]+\mathbf{P}[k] \mathbf{H}^{T} \Sigma_{v}^{-1}\left(-a_{m}^{\text {body }}[k]-(\mathbf{H} \bar{z}[k])\right)
$$

where

$$
\mathbf{P}[k]=\mathbf{M}[k]-\mathbf{M}[k] \mathbf{H}^{T}\left(\mathbf{H M}[k] \mathbf{H}^{T}+\boldsymbol{\Sigma}_{v}\right)^{-1} \mathbf{H M}[k]
$$

model update (time update):

$$
\begin{aligned}
\bar{z}[k+1] & =\mathbf{F} \hat{z}[k]+\mathbf{G} a_{m}^{\text {body }}[k] \\
\mathbf{M}[k+1] & =\mathbf{F} \mathbf{P}[k] \mathbf{F}^{T}+\mathbf{G}_{1} \boldsymbol{\Sigma}_{w} \mathbf{G}_{1}^{T} .
\end{aligned}
$$

One-hour-long motionless sensor data were collected to estimate the covariances $\boldsymbol{\Sigma}_{w}$ and $\boldsymbol{\Sigma}_{v}$, so that $w$ and $v$ would approximately be white.

Finally, the estimated bias $a_{b}^{\text {body }}$ is transformed to be represented in the navigation coordinate frame and then subtracted from the measured acceleration to get the actual acceleration in the navigation frame, as given by

$$
a_{a}^{\text {nav }}[k]=a_{m}^{\text {nav }}[k]-\mathbf{R}_{\mathrm{nb}}[k] a_{b}^{\text {body }}[k] .
$$

The output of the KF is plotted in Fig. 9. With this extra level of filtering, the final position error was decreased by about $5 \%-10 \%$ in most of our tests. Positions that are calculated 
Position Calculated from IMU's Gravity-Compensated Data (w/ZUPT'ing)

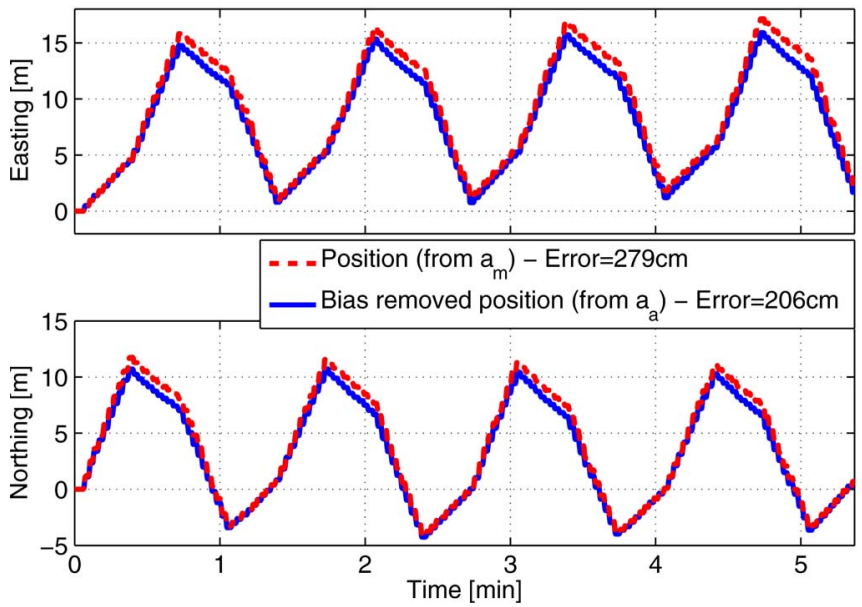

Fig. 10. Comparison of two positions calculated from measured and actual accelerations using the ZUPT'ing scheme. Easting refers to the movement toward the east, and Northing refers to the movement toward the north.

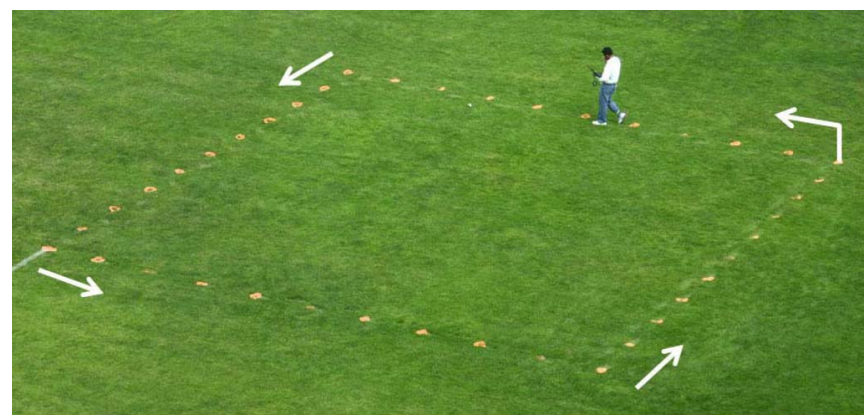

Fig. 11. Calibration step points around the square calibration loop.

from measured and actual (bias compensated) accelerations are compared in Fig. 10. For the case shown in this figure, the loopclosing error was decreased by $26 \%$.

\section{B. Calibration}

In initial loop-closing tests, we realized that our position results have drifts toward a fixed direction. We believe that this is due to an error in the IMU's internal gravity compensation algorithm. To cancel this random drift effect, we developed a calibration scheme that was applied each time that we performed an experiment. We marked 40 points around an 11.7-m/edge square in the field such that the user can walk on these marked step points. Walking with uniform steps, the user completed four to six laps (Fig. 11) around the square. Using these initial data, 40 lines were fitted through the position of the same step points (Fig. 12). An average slope was determined from the linear fits, and the slope was used to correct the deviation in the future collected data. A developed calibration scheme was found to be user-independent but depended on the sensors that were used.

\section{Walk Experiments}

We performed loop-closing half-hour walks to test our system. The procedure that we followed is depicted in Fig. 13. First, we performed a 5-min short walk to be used in the

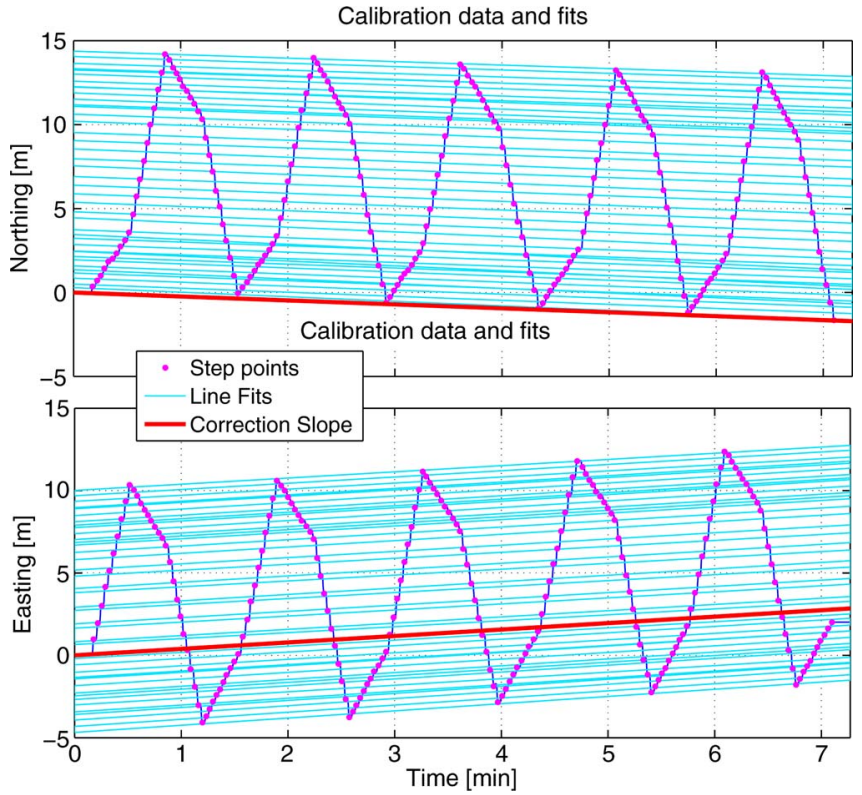

Fig. 12. Linear fits are shown for the steps during the calibration walk. The average slope of the linear fits was used to correct the deviation in the future data. Easting refers to the movement toward the east, and Northing refers to the movement toward the north.

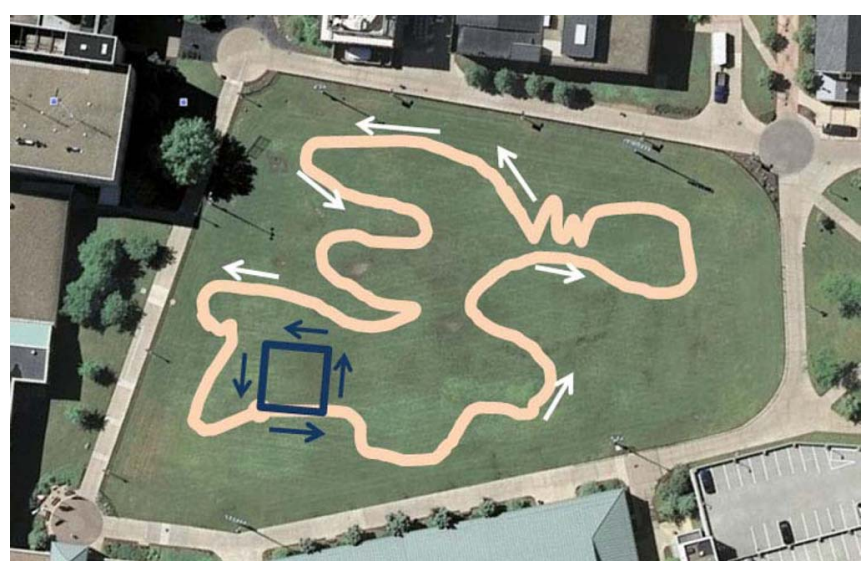

Fig. 13. Procedure for loop-closing half-hour walks. 1) Short walk around a square (11.7 m/edge) for $5 \mathrm{~min} .2$ ) Longer half-hour walks around the same square as a short walk or a random path (paths are shown for illustrative purposes only).

calibration process described in the previous section. Next, we performed longer half-hour walks ending at the starting point to calculate the loop-closing error for each walk.

The field where we performed our walk tests is a sports field with a reasonably flat surface, and we are assuming that the person is walking on a horizontal surface. Therefore, the position errors reported in this paper are the Euclidean norm of the 2-D errors. We have conducted six half-hour loop-closing experiments; the subject first walked along the square-shaped path for about $5 \mathrm{~min}$ for the calibration. Then, we collected additional half-hour walk data either around the same square as the calibration walk or a random path in the field.

\section{Walk Results}

Figs. 14 and 15 show two of the walk results. "Position Error" is calculated by integrating the measured acceleration $a_{m}$ 


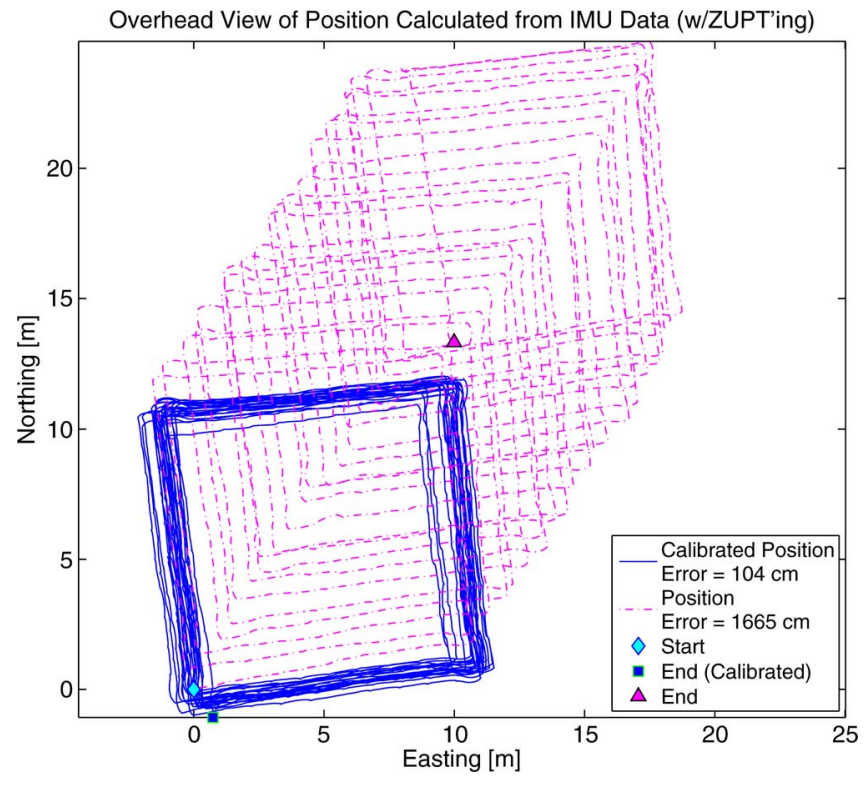

Fig. 14. Half-hour square loop walk results. Position is calculated by integrating the measured acceleration $a_{m}$ with ZUPT'ing, and Calibrated Position is calculated from integrated actual acceleration $a_{a}$ with ZUPT'ing followed by the calibration. Loop-closing error is $1.04 \mathrm{~m}(0.098 \%)$. Easting refers to the movement toward the east, and Northing refers to the movement toward the north.

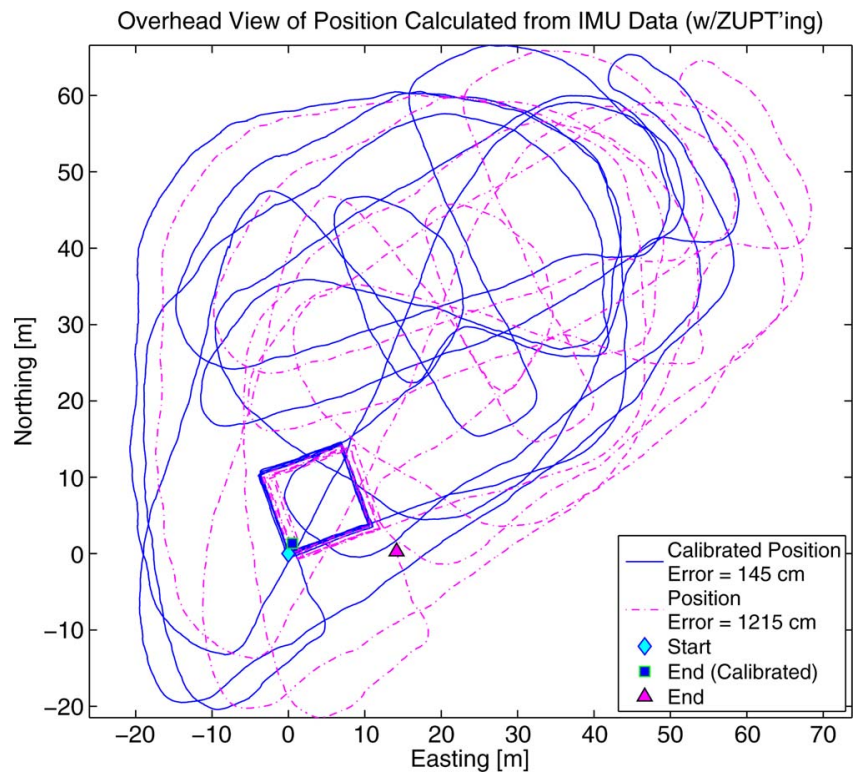

Fig. 15. Half-hour random walk results. Position is calculated by integrating the measured acceleration $a_{m}$ with ZUPT'ing, and Calibrated Position is calculated from integrated actual acceleration $a_{a}$ with ZUPT'ing followed by the calibration. Loop-closing error is $1.45 \mathrm{~m}(0.078 \%)$. Easting refers to the movement toward the east, and Northing refers to the movement toward the north.

with ZUPT'ing, and “Calibrated Position Error” is calculated from integrated actual acceleration $a_{a}$ with ZUPT'ing followed by the calibration. Fig. 14 shows a walk experiment where the user followed the square loop for the whole experiment. Loop-closing error for this experiment is $1.04 \mathrm{~m}(0.098 \%)$. This result shows the effectiveness of the calibration process that was performed. Fig. 15 shows a calibration walk followed by

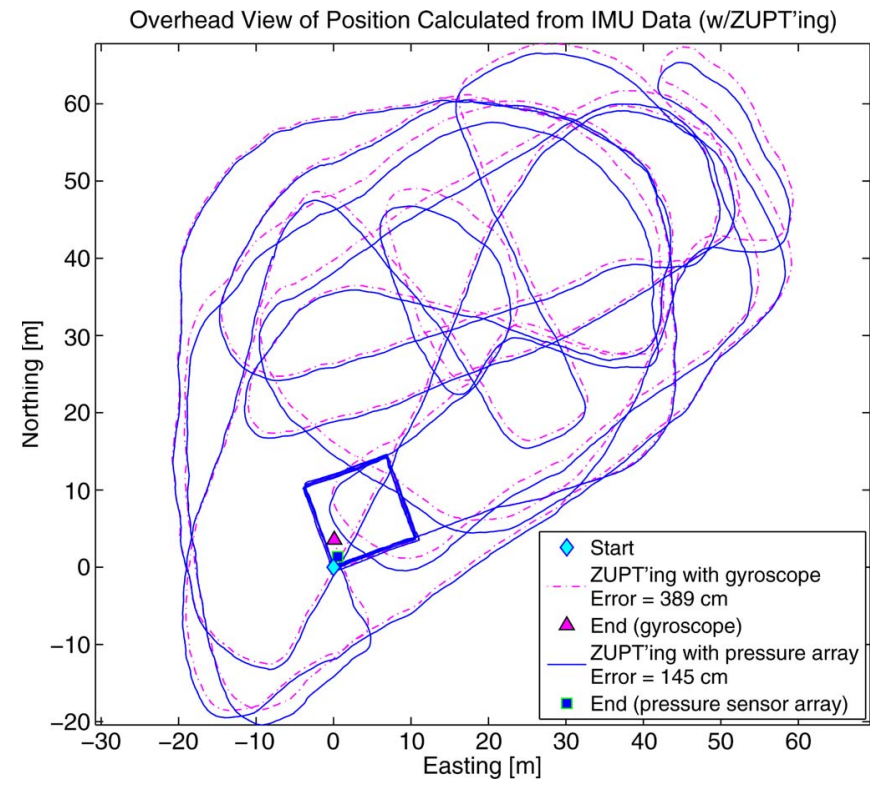

Fig. 16. Half-hour random walk results. Loop-closing error is $1.45 \mathrm{~m}$ $(0.078 \%)$ for ZUPT'ing with the PPS pressure sensor array's zero-velocity points and $3.89 \mathrm{~m}(0.21 \%)$ for ZUPT'ing with the gyroscope's zero-velocity points. Easting refers to the movement toward the east, and Northing refers to the movement toward the north.

TABLE I

EXPERIMENTAL RESULTS

\begin{tabular}{|c|c|c|c|}
\hline $\begin{array}{c}\text { Exp } \\
\text { No }\end{array}$ & Distance $[\mathrm{m}]$ & Gyroscope [m] & Pressure Sensor [m] \\
\hline 1 & 987 & $1.97(0.20 \%)$ & $2.96(0.30 \%)$ \\
\hline 2 & 656 & $4.98(0.76 \%)$ & $6.14(0.94 \%)$ \\
\hline 3 & 930 & $6.45(0.69 \%)$ & $4.90(0.53 \%)$ \\
\hline 4 & 1056 & $2.52(0.24 \%)$ & $1.04(0.098 \%)$ \\
\hline 5 & 1848 & $3.89(0.21 \%)$ & $1.45(0.078 \%)$ \\
\hline 6 & 1811 & $9.39(0.52 \%)$ & $9.33(0.52 \%)$ \\
\hline Mean & 1215 & $4.87 \pm 2.75(0.40 \%)$ & $4.30 \pm 3.15(0.35 \%)$ \\
\hline
\end{tabular}

Same ZUPT'ing and calibration techniques are applied to calculate loop-closing position errors (and percent relative errors) using zero-velocity points provided from the two sensors.

a random walk experiment in the field. Loop-closing error for this experiment is $1.45 \mathrm{~m}(0.078 \%)$.

The average path length walked during half-hour walks was $1215 \mathrm{~m}$ (this is in addition to an average of 235-m calibration walks). We have conducted six walk experiments, and the loopclosing errors have a mean of $4.30 \pm 3.15 \mathrm{~m}$ (mean \pm standard deviation), which makes the average relative error $0.35 \%$.

It is also possible to get the zero-velocity information from the IMU's integrated accelerometer or gyroscope. We compared the position results obtained with ZUPT'ing using zero-velocity points provided from the IMU and those of the pressure sensor array. In our tests, we found that the IMU's gyroscope can be used to detect zero-velocity points; therefore, angular velocity was used to detect the zero-velocity of the user. In Fig. 16, two walk paths that are calculated using the zero-velocity points of the pressure sensor and the gyroscope are shown. The same ZUPT'ing and calibration techniques are applied. For the walk 
TABLE II

Design Goals AND MeAsured Results

\begin{tabular}{|l|c|c|c|}
\hline System Metric & State of the art & Goal & Results \\
\hline Navigation accuracy for $1 / 2$ hour walk $[\mathrm{m}]$ & 45 & $\leq 10$ & $4.30 \pm 3.15$ \\
\hline Zero-velocity sensing bias per step $[\mathrm{mm} / \mathrm{s}]$ & 28 & $\leq 4$ & 4 (imaging limited) \\
\hline Maximum volume for velocity sensor alone $\left[\mathrm{cm}^{3}\right]$ & 125 & $\leq 300$ & 5 \\
\hline Power consumption of the velocity sensor $[\mathrm{mW}]$ & 2000 & 10 & 20 \\
\hline Number of GRSC sensor elements & 10 COTS) & $24(\mathrm{COTS})$ \\
\hline
\end{tabular}

shown in the figure, the pressure sensor's zero-velocity points provide two times better accuracy than the gyroscope's zerovelocity points.

If the gyroscope's zero-velocity points were used in ZUPT'ing instead of those of the pressure sensor, the mean loop-closing error for all the half-hour walks would be $4.87 \pm$ $2.75 \mathrm{~m}$. The experimental results are summarized in Table I. When the two means are compared, an average of $12 \%$ improvement in the end-position accuracy was obtained with the use of a biomechanical sensor in the developed micronavigation system. Although the difference between these two data sets is not statistically significant, we believe that with more data points, statistical significance can be shown.

\section{CONCLUSION}

In this paper, we have presented a personal micronavigation system that uses inertial measurements from an IMU and zerovelocity measurements from a GRSC. Our design goals, specifications from state of the art, and results from the developed system are given in Table II. All of the design goals have been achieved. Only the zero-velocity sensing bias goal has been reached indirectly. That is due to the placement of the pressure sensor array in the shoe. The closer the sensor is to the ground, the better the detection of zero-velocity regions gets. Although the pressure sensor array in our setup has been placed under the insole of the shoe, we have been able to effectively observe the zero-velocity points using pressure contour velocities. Also, we have assumed acceleration bias to be invariable, but such a scheme can also adapt to slowly drifting bias values due to the nature of the KF.

Using a higher density pressure sensor array is a possible way to further improve the system. As the number of the pressure sensor elements increases, the resolution of the pressure contours increases, and with that, the minimum detectable velocity becomes closer to zero. This would improve the performance of ZUPT'ing. We also would like to note that, in this paper, only the loop-closing errors have been used to verify the system accuracy. A differential GPS (DGPS) could be used to measure the accuracy of the system throughout the trajectory. Our straight-walk and around-the-square-loop experiments have shown that we did not have any other form of error that would not be shown by the loop-closure check, but would be visible in the DGPS data.

Finally, our walking tests have been carried out on the surfaces that provided uniform foot reaction at all times. Additional testing of the pressure sensor array and algorithm development is necessary for surfaces such as gravel, rocks, sand, mud, and snow. This is beyond the scope of this paper at this stage of development.

\section{REFERENCES}

[1] J. Kim, J.-G. Lee, G.-I. Jee, and T. K. Sung, "Compensation of gyroscope errors and GPS/DR integration," in Proc. IEEE Position Location Navigation Symp., Apr. 1996, pp. 464-470.

[2] Z. Zhou, X. Chen, and T. Zhang, "The principle of non-sensor dead reckoning," in Proc. IEEE Geosci. Remote Sens. Symp., Jul. 2005, vol. 7, pp. 4693-4696.

[3] W.-W. Kao, "Integration of GPS and dead reckoning navigation systems," in Proc. IEEE Vehicle Navigation Inf. Syst. Conf., Oct. 1991, vol. 2, pp. 635-643.

[4] E. S. Sazonov, T. Bunpus, S. Zeigler, and S. Marocco, "Classification of plantar pressure and heel acceleration patterns using neural networks," in Proc. IEEE Neural Netw. Conf., Jul./Aug. 2005, vol. 5, pp. 3007-3010.

[5] M. Lord and R. Hosein, "Pressure distribution by molded inserts in diabetic footwear: A pilot study," J. Rehab. Res. Develop., vol. 31, no. 3, pp. 214-221, Aug. 1994.

[6] C. Randell, C. Djiallis, and H. Muller, "Personal position measurement using dead reckoning," in Proc. IEEE Int. Symp. Wearable Comput., Oct. 2005, pp. 166-173.

[7] L. Fang, P. J. Antsaklis, L. Montestruque, M. B. McMickell, M. Lemmon, Y. Sun, H. Fang, I. Koutroulis, M. Haenggi, M. Xie, and X. Xie, "Design of a wireless assisted pedestrian dead reckoning system-The NavMote experience," IEEE Trans. Instrum. Meas., vol. 54, no. 6, pp. 2342-2358, Dec. 2005.

[8] L. Ojeda and J. Borenstein, "Non-GPS navigation for emergency responders," in Proc. Int. Joint Topical Meeting: Sharing Solutions Emergencies Hazardous Environ., Salt Lake City, UT, Feb. 2006.

[9] L. Ojeda and J. Borenstein, "Non-GPS navigation with the personal dead reckoning system," in Proc. SPIE Defense Security Conf., Unmanned Syst. Technol. IX, Orlando, FL, Apr. 2007, pp. 1-11.

[10] L. Ojeda and J. Borenstein, "Non-GPS navigation for security personnel and emergency responders," J. Navigation, vol. 60, no. 3, pp. 391-407, Sep. 2007.

[11] S. Godha and G. Lachapelle, "Foot mounted inertial system for pedestrian navigation," Meas. Sci. Technol., vol. 19, no. 7, pp. 075 202-1-075 202-9, Jul. 2008 .

[12] J. Borenstein, L. Ojeda, and S. Kwanmuang, "Heuristic reduction of gyro drift for personnel tracking systems," J. Navigation, vol. 62, no. 1, pp. 4158, Jan. 2009.

[13] J. Cheung, M. Zhang, A. Leung, and Y. Fan, "Three-dimensional finite element analysis of the foot during standing-A material sensitivity study," J. Biomech., vol. 38, no. 5, pp. 1045-1054, May 2005.

[14] M. Trew and T. Everett, Human Movement: An Introductory Text, 4th ed. New York: Elsevier Health Sciences, 2001.

[15] H. Lanshammar and L. Strandberg, "Horizontal floor reaction forces and heel movements during the initial stance phase," in Biomechanics VIII-B. Champaign, IL: Human Kinetic, 1982, pp. 1123-1128.

[16] T. Judd, "A personal dead reckoning module," in Proc. Inst. Navigation Global Positioning Syst. Conf., Kansas City, MO, Sep. 1997.

[17] M. Kourogi and T. Kurata, "Personal positioning based on walking locomotion analysis with self-contained sensors and a wearable camera," in Proc. 2nd IEEE/ACM ISMAR, Oct. 2003, pp. 103-112.

[18] R. Jirawimut, P. Ptasjnski, V. Garaj, F. Cecelja, and W. Balachandran, "A method for dead reckoning parameter correction in pedestrian navigation system," in Proc. IEEE Instrum. Meas. Technol. Conf., Budapest, Hungary, May 2001, pp. 1554-1558. 
[19] J. Elwell, "Inertial navigation for the urban warrior," Proc. SPIE—Int. Soc. Opt. Eng., vol. 3709, pp. 196-204, 1999.

[20] R. Stirling, J. Collin, K. Fyfe, and G. Lachapelle, "An innovative shoemounted pedestrian navigation system," in Proc. Eur. Navigation Conf. (GNSS), Graz, Austria, Apr. 22-25, 2003, pp. 103-112.

[21] S. Y. Cho and C. G. Park, "MEMS based pedestrian navigation system," J. Navigation, vol. 59, no. 1, pp. 135-153, 2006.

[22] J. Saarinen, J. Suomela, S. Heikkila, M. Elomaa, and A. Halme, "Personal navigation system," in Proc. IEEE/RSJ IROS, 2004, vol. 1, pp. 212-217.

[23] T. Brand and R. Phillips, "Foot-to-foot range measurement as an aid to personal navigation," in Proc. 59th Inst. Navigation Annu. Meeting, Albuquerque, NM, 2003.

[24] R. Feliz, E. Zalama, and J. G. Garcia-Bermejo, "Pedestrian tracking using inertial sensors," J. Phys. Agents, vol. 3, no. 1, pp. 35-42, Jan. 2009.

[25] E. Foxlin, "Pedestrian tracking with shoe-mounted inertial sensors," IEEE Comput. Graph. Appl., vol. 25, no. 6, pp. 38-46, Nov./Dec. 2005.

[26] Eagle Digital RealTime System, Motion Analysis Corporation, Santa Rosa, CA, 2009. [Online]. Avaialble: http://www.motionanalysis.com/ $\mathrm{html} /$ movement/eagle.html

[27] Pedar System, Pressure Insole Size XW, Novel Electronics Incorporated, Munich, Germany, 2009. [Online]. Available: http://www.novelusa.com/ index.php?fuseaction $=$ systems.pedar

[28] Product Manual for Use With InterSense InertiaCube3 and the InterSense InertiaCube Processor, 2nd ed., InterSense, Inc., Bedford, MA, 2007. [Online]. Available: www.intersense.com/products/downloads/ InertiaCube3\%5FManual.pdf

[29] G. F. Franklin, J. D. Powell, and M. Workman, Digital Control of Dynamic Systems, 3rd ed. Reading, MA: Addison-Wesley, 1998.

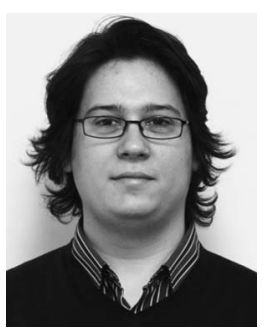

Özkan Bebek (S'06-M'09) received the B.S. degree in mechanical engineering from Middle East Technical University, Ankara, Turkey, in 2001, the M.S degree in mechatronics from Sabanci University, Istanbul, Turkey, in 2003, and the Ph.D. degree in systems and control engineering from Case Western Reserve University, Cleveland, $\mathrm{OH}$, in 2008.

$\mathrm{He}$ is currently a Postdoctoral Research Associate with the Department of Electrical Engineering and Computer Science, Case Western Reserve University. His research interests include medical robotics, mechatronics, biomedical measurement, control systems, and haptics.

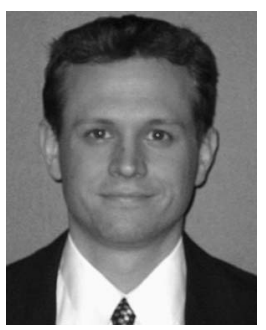

Michael A. Suster (S'06) received the B.S. and M.S degrees in electrical engineering in 2002 and 2004, respectively, from Case Western Reserve University, Cleveland, $\mathrm{OH}$, where he is currently working toward the Ph.D. degree in electrical engineering.

His research interests include low-noise analog integrated circuit design for MEMS sensor interface electronics and wireless integrated sensing microsystems.

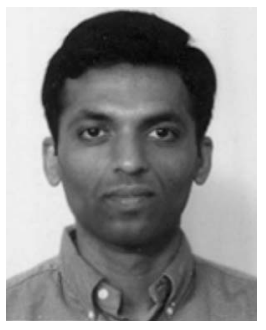

Srihari Rajgopal (S'01) received the M.S. degree in electrical engineering and applied physics, in the field of microelectromechanical systems, from Case Western Reserve University, Cleveland, OH, in 1999.

From 1999 to 2003, he was employed in various microsystem-related research capacities, first at Xactix, Pittsburgh, PA, and then at FLXMicro, Cleveland. Since 2003, he has been with Prof. Mehregany's group in the Department of Electrical Engineering and Computer Science, Case Western Reserve University, where he is currently an Engineer. Since September 2009, he has held the additional appointment of Adjunct Lecturer in the same department. He has authored or coauthored several publications covering research in devices and device integration, including microvalves, flow sensors, inertial sensors, pressure sensors, and deep brain stimulation electrodes, as well as process development in silicon carbide ( $\mathrm{SiC})$. $\mathrm{He}$ has also coauthored a book chapter on microsystem reliability. His current research interests include the development of reactive ion etch techniques for $\mathrm{SiC}$ and the development of piezoresistive $\mathrm{SiC}$-based microsystems. He is a Reviewer for the Journal of Microelectromechanical Systems.

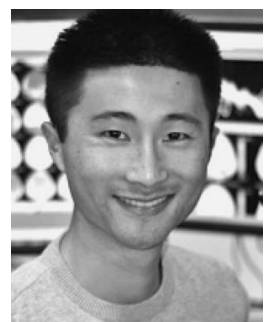

Michael J. Fu (S'06) received the B.S. degree in electrical engineering and computer science in 2003 from the University of California, Berkeley, and the M.S. degree in electrical engineering in 2006 from Case Western Reserve University (CWRU), Cleveland, $\mathrm{OH}$, where he is currently working toward the Ph.D. degree in the Department of Electrical Engineering.

In 2007, he was a Graduate Student Fellow with the National Aeronautics and Space Administration Lewis' Educational and Research Collaborative Internship Project. His current research interests include haptics, medical robotics, and rehabilitation engineering.

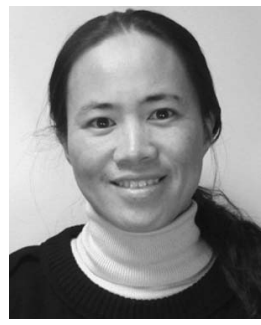

Xuemei Huang received the B.S. degree in marine chemistry from Ocean University of Qingdao, Qingdao, China, in 1994, the M.S. degree in analytical chemistry from Nankai University, Tianjin, China, in 1997, and the M.S. degree in biomedical engineering from Case Western Reserve University, Cleveland, $\mathrm{OH}$, in 2002.

Since 2001, she has been a Research Engineer with the Department of Biomedical Engineering, Lerner Research Institute, Cleveland Clinic, Cleveland. Her current work involves biomechanics modeling of human movements.

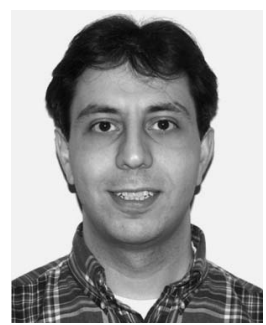

M. Cenk Çavuşoğlu (S'93-M'01-SM'06) received the B.S. degree in electrical and electronic engineering from Middle East Technical University, Ankara, Turkey, in 1995 and the M.S. and Ph.D. degrees in electrical engineering and computer sciences from the University of California (UC), Berkeley, in 1997 and 2000, respectively.

From 2000 to 2002, he was a Postdoctoral Researcher and a Lecturer with the Department of Electrical Engineering and Computer Sciences, UC Berkeley. He is currently an Associate Professor of electrical engineering and computer science with Case Western Reserve University, Cleveland, $\mathrm{OH}$, and a Visiting Associate Professor with the Department of Electrical and Electronic Engineering, Bilkent University, Ankara. His research involves applications of robotics and control engineering to biomedical and biologically inspired engineered systems. Specifically, his research interests include robotics, systems and control theory, and human-machine interfaces, with emphasis on medical robotics, haptics, virtual environments, surgical simulation, and biosystem modeling and simulation.

Dr. Çavuşoğlu served as an Associate Editor of the IEEE TRANSACTIONS ON RoBOTICs between 2005 and 2009.

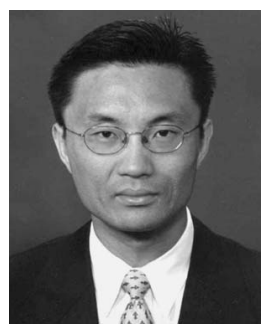

Darrin J. Young (S'93-M'99) received the B.S., M.S., and Ph.D. degrees from the University of California, Berkeley, in 1991, 1993, and 1999, respectively.

In 1999, he joined the Department of Electrical Engineering and Computer Science, Case Western Reserve University, Cleveland, $\mathrm{OH}$, as an Assistant Professor. In 2009, he joined the Department of Electrical and Computer Engineering, University of Utah, Salt Lake City, as a Utah Science and Technology Research Initiative Associate Professor. He has published many technical papers in journals and conferences. His research interests include microelectromechanical system design, fabrication, and integrated analog circuit design for wireless sensing, biomedical implant, communication, and general industrial applications.

Dr. Young is an Associate Editor of the IEEE Journal of Solid-State Circuits and the Chair of the IEEE Electron Devices Society Microelectromechanical Systems Committee. He has served as a technical program committee member and the session chair for a number of international conferences. 


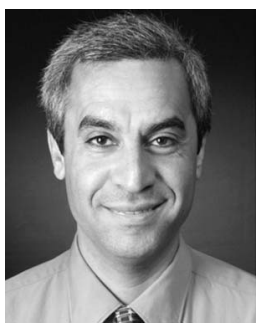

Mehran Mehregany (S'88-M'92-SM'00) received the M.S. and Ph.D. degrees in electrical engineering from the Massachusetts Institute of Technology, Cambridge, in 1986 and 1990, respectively.

From 1986 to 1990 , he was a Consultant with the Robotic Systems Research Department, AT\&T Bell Laboratories, where he was a key contributor to groundbreaking research in microelectromechanical systems (MEMS). In 1990, he joined the Department of Electrical Engineering and Applied Physics, Case Western Reserve University, Cleveland $\mathrm{OH}$, as an Assistant Professor. In 1997, he was promoted to Full Professor, in 1998 to 2000, he held the George S. Dively Professor of Engineering Endowed Chair, and he has since held the Goodrich Professor of Engineering Innovation Endowed Chair. From 1995 to 2002, he served as the Director of the MEMS Research Center, and, from January 2003 to January 2006, he was the Chairman of the Department of Electrical Engineering and Computer Science. He is well known for his research in the area of MEMS and silicon carbide. From January 1996 to December 1997, he was the Editor-in-Chief of the Journal of Micromechanics and Microengineering. He has over 300 publications describing his work. He is the holder of 18 U.S. patents. His current research interests include the intersections of microelectromechanical/nanoelectromechanical systems, semiconductor silicon carbide, and integrated circuits for a wide range of applications.

Dr. Mehregany was a recipient of a number of awards/honors and has founded several technology startups. From 1994 to 2004, he served as the Assistant-to-the-President of the Transducers Research Foundation, where he currently serves as a Trustee and the Treasurer.

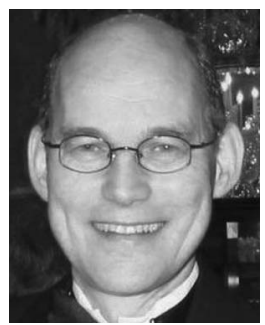

Antonie J. (Ton) van den Bogert received the B.S. and M.S. degrees in physics and applied mathematics and the Ph.D. degree in veterinary sciences for computational modeling of locomotion in horses in 1989 from the University of Utrecht, Utrecht, The Netherlands.

He was a Postdoctoral Researcher in sports biomechanics with the University of Calgary, Calgary, AB, Canada, where, in 1993, he became a Faculty Member. In 1998, he moved to Cleveland, where he is currently an Associate Staff with the Lerner Research Institute, Cleveland Clinic, and an Associate Professor with Case Western Reserve University. He has published over 90 journal articles and book chapters, and sits on the editorial boards of four journals. His current research interest includes human biomechanics and motor control, with applications in the prevention and rehabilitation of musculoskeletal injuries and the development of neural and mechanical prosthetics technology. This work is supported by innovative methods for analysis of human movement and computational modeling of musculoskeletal system dynamics.

Dr. van den Bogert has been the Moderator of the e-mail discussion group BIOMCH-L since 1988, which has more than 6000 subscribers. He has been active in the International Society of Biomechanics as a Cofounder and the Chair of the Technical Group Computer Simulation and a member of the Executive Board. He is currently the society's President-Elect. He was a recipient of the O'Donoghue Sports Injury Research Award from the American Orthopaedic Society for Sports Medicine in 2004 and of the Technical Achievement Award from the Academy of Motion Picture Arts and Sciences in 2005.

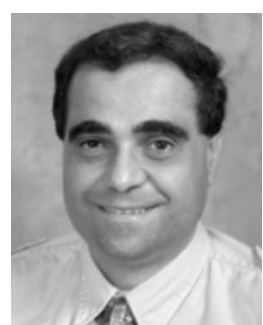

Carlos H. Mastrangelo (S'84-M'90) was born in Buenos Aires, Argentina, in 1960. He received the B.S., M.S., and Ph.D. degrees in electrical engineering and computer science from the University of California, Berkeley, in 1985, 1988, and 1991, respectively.

His graduate work concentrated on the applications of microbridges in microsensor technology. From 1991 to 1992, he was with the Scientific Research Laboratory, Ford Motor Company, Dearborn, MI, developing microsensors for automotive applications. From 1993 to 2002, he was an Associate Professor of electrical engineering and computer science with the Center for Integrated Microsystems, University of Michigan, Ann Arbor. From 2000 to 2005, he was the Vice President of Engineering with Corning-Intellisense, Wilmington, MA, and the Director of the Biochemical Technologies Research Group, Corning, NY. From 2005 to 2008, he was an Associate Professor of electrical engineering and computer science with Case Western Reserve University, Cleveland, $\mathrm{OH}$. $\mathrm{He}$ is currently a Utah Science and Technology Research Initiative Professor of electrical engineering and bioengineering with the University of Utah, Salt Lake City. His research focuses on microelectromechanical system applications and technology, microfluidic systems, and integration, design, and modeling of MEMS fabrication processes.

Dr. Mastrangelo is on the editorial boards of Sensors and Actuators and the IEEE/ASME Journal of Microelectromechanical Systems. He has participated in technical and organizing committees of numerous International Society for Optical Engineers and IEEE conferences in the MEMS area. 